\title{
Salience: Agenda Choices by Competing Candidates*
}

\author{
Marcus Berliant ${ }^{\dagger} \quad$ Hideo Konishi ${ }^{\ddagger}$
}

May 19, 2004

\begin{abstract}
Which issues are discussed by candidates in an election campaign? Why are some issues never discussed? Model tractability is lost quickly when dealing with these questions, partly because of the multidimensional voting inherent in models of multiple issues. Our model features two candidates for office who can talk about any subset of issues, allowing uncertainty both on the part of voters and candidates, and taking candidates to be office motivated. Candidates move first and simultaneously, announcing any positions they choose on any issues. To us, salience is simply the discussion of an issue in a campaign. If both candidates and voters are expected utility maximizers, we find salience results, in that candidates typically want to talk about everything (or they are indifferent between talking and nonsalience). Leaving the expected utility framework, we present an example using "Knightian un-
\end{abstract}

${ }^{*}$ The authors thank an anonymous referee, Sophie Bade, Ken Binmore, Steve Callander, John Duggan, Matt Jackson, John Ledyard, Eric Rasmusen, seminar participants at the Wallis Institute of the University of Rochester, at Stanford University, at Caltech, at the Public Choice Society Conference in San Diego, and at the Midwest Economic Theory and International Trade Conference in Bloomington for helpful comments, and Clara Asnes for help with the figures, but retain responsibility for any errors. The first author gratefully acknowledges financial support in the form of a fellowship from the American Philosophical Society. The second author gratefully acknowledges financial support in the form of a fellowship from the Japan Society for the Promotion of Science.

${ }^{\dagger}$ Department of Economics, Washington University, Campus Box 1208, One Brookings Drive, St. Louis, MO 63130-4899, USA, berliant@wueconc.wustl.edu; Division of the Humanities and Social Sciences, California Institute of Technology

${ }^{\ddagger}$ Department of Economics, Boston College, 140 Commonwealth Avenue, Chestnut Hill, MA 02467-3806, USA 
certainty" or "maxmin expected utility with multiple priors" of GilboaSchmeidler to illustrate how robust nonsalience and salience of issues might be generated. 


\section{Introduction}

Which issues are discussed by candidates in an election campaign? Why are different issues discussed by different candidates at the same time? Why are some issues never discussed? The last question is of particular interest in political science. It seems that there is no consensus about how nonannouncement of a position (nonsalience) on an issue can occur. In this paper, we attempt to examine what conditions are needed to demonstrate nonsalience of an issue.

To us, salience is simply the discussion of an issue in a campaign. Partial salience of an issue means that one candidate talks about it, while salience means that both candidates talk about it. Nonsalience means that neither candidate discusses it.

Nonsalience is observed in many elections. It is typical that presidential candidates talk about irrelevant issues such as education reform plans (though the federal government has no power to control education), while they do not discuss important and sensitive issues such as gun control policies.

Our informal explanation for nonsalience is as follows. Suppose that two candidates are running for election. They can freely choose issues (agendas) to discuss, and can choose their positions on these issues. Do they want to announce their positions on all issues? There may be some issues (such as gun control) over which the voters' preference distribution is very uncertain (candidates cannot predict accurately what portion of voters are for the policy, and how strongly they feel about the policy), since these issues have not been discussed in past elections. Announcing positions on such issues can be dangerous. If voters happen to be against a candidate's announced position and they feel very strongly about the issue, then she may lose the election only because she announced a position on this issue. Thus, if candidates are risk (ambiguity)-averse, then such issues may not be discussed by either candidate and a nonsalience result applies.

It is not an easy task to formalize this idea. Model tractability is lost quickly when dealing with these questions, partly because of the multidimensional voting inherent in models of multiple issues. But there are more problems. First, in order to preclude trivial results where all candidates announce (the ex ante winning) positions on all issues immediately, it seems necessary to construct a model where there is both uncertainty on the part of the voters concerning where the candidates stand in the absence of an announcement, and where the candidates are uncertain about the distribution of voters. Second, it would be easier but much less natural from the standpoint of politics to 
construct a model where candidates and voters move simultaneously, possibly using mixed strategies (particularly for the candidates). Once we allow the candidates to announce simultaneously their positions first (or announce no position on an issue), followed by realizations of their mixed strategies or coin flips, followed by voting, we run into discontinuous payoffs and games.

To bypass at least some of these issues, we make the simple assumption that there are two candidates or parties who are completely motivated by holding office. This allows us some tractability, in the form of a constant sum game between the two candidates in the first stage, while limiting any statements we can make about the policy content for candidates of the issues discussed (though it still allows policy to matter for voters). Rather, we focus on the relationship between risk and the issues discussed in a campaign. Even then, tractability is not easy, and it is difficult to obtain nonsalience. Candidates want to discuss everything.

Five papers we have found in the literature related to our work are Shepsle (1972), Glazer (1990), Alesina and Cukierman (1990), Adams (1999), and Glazer and Lohmann (1999). Shepsle (1972) is generally considered the seminal paper on the topic of candidate ambiguity in elections. He analyzes a two candidate model in which the policy space is one-dimensional and each voter has a bliss point in the space. Candidates are office-motivated. They are assumed to be asymmetric in the sense that one is an incumbent and the other is a challenger. The incumbent is required to announce her position, while the challenger is assumed to announce her position ambiguously: a probability distribution over policy positions. Thus, voters face uncertainty over the challenger's policy, and they vote according to their expected utilities. Shepsle shows that the challenger can win only when voters are risk-lovers (see also Aragones and Postlewaite, 2002). Although Shepsle does not talk about salience, his model can be interpreted as a model that generates a nonsalience outcome by assuming that both candidates can choose not to announce their policies, and that voters have subjective beliefs over candidates' policy positions. Nonsalience may occur when voters are risk lovers.

This early literature, including McKelvey (1980), had two features in common. First, the essential intuition of the results are clear. If a candidate is employing an ambiguous strategy, then replacing it with the mean of the ambiguous distribution over that candidate's position as seen by the median voter will (second order) stochastically dominate the ambiguous strategy, leading to higher utility of the median voter under risk aversion. Thus, salience is 
a better strategy for the candidate. Second, explicit in this work and implicit in this argument is that the candidates know voter preferences, and face no uncertainty.

The more modern literature relaxes this last assumption. Is nonsalience (ambiguity) possible in equilibrium when candidates are uncertain about voter policy preferences? One of our main results below shows that with multiple issues and even risk neutral voters, ambiguity is not a robust equilibrium strategy. If one wants to generate robust ambiguity and robust salience each as equilibrium outcomes so that comparative statics can be derived and tested, some standard assumption must be relaxed. We relax the expected utility hypothesis for the candidates. Glazer (1990), clearly the model closest to ours, relaxes the assumption that the candidate knows the mean of the median voter's distribution over candidate policy outcomes under the ambiguous strategy. Alesina and Cukierman (1990) assume that policy matters to candidates (or parties) in addition to holding office. We discuss these two papers and their relation to our work next.

Glazer (1990) finds that for a set of parameter values with nonempty interior, salience is the equilibrium strategy of candidates and for another set of parameter values with nonempty interior, nonsalience is the equilibrium strategy. As his results are informal and in conflict with ours, we have tried in Appendix 2 to make one of his examples formal by placing it in a Bayesian game framework. Although the risk aversion of the median voter is highlighted in this paper, it is not as far as we can tell the proximate cause of the difference in results. Rather, it is the assumption in this paper that the candidates are lost in space (specifically, the integers), in that if they announce a position, they do not have much of an idea about where it is relative to the mean of the median voter's guess about where the candidate's policy will be under ambiguity. In contrast, we assume that any candidate's distribution over policy positions with no announcement is common knowledge. In our view, the latter assumption is more persuasive, since what voters think about a candidate's stance on an issue that she hasn't discussed can be found by surveying the voters. Even if one rejects this view, we have put the Glazer analysis into a game-theoretic setting and made this implicit assumption clear.

Alesina and Cukierman (1990) develop a two period model with two sided uncertainty where candidates have policy preferences in addition to office motivation. In equilibrium, the incumbent might wish to take an ambiguous policy position (while in office) to cloud his true policy preferences in case 
they deviate from those of the median voter. Without policy preferences, they state explicitly (p. 841) that the equilibrium degree of ambiguity chosen by the incumbent is zero. The insights from this model seem highly dependent on the functional forms used. ${ }^{1}$

These are, of course, three distinct theories of ambiguity. Which one is correct in various circumstances is an empirical question. Naturally, one must first specify the theories in order to test them.

A fourth theory, proposed by a referee, is that candidates face time and money constraints in exposing their positions, so the candidates optimize subject to these constraints, and thus the constraints limit the number of issues discussed. This type of theory would be useful in addressing how many issues are discussed in an election rather than which issues are discussed or whether the candidates discuss any issues at all. Clearly, it would be interesting but complicated to graft the budget model onto the models we have reviewed.

Next we discuss more recent but less closely related literature.

Adams (1999) features a probabilistic voting framework where parties (the equivalent of our candidates) are vote or rank maximizing. Salience of an issue is embedded in all voters' utility functions via a weight on that issue. The main theoretical result is that parties adopt the most popular platform. This model is not set up to address the kinds of questions we discuss.

Glazer and Lohmann (1999) features two candidates and one voter, all of whom are policy driven. The only decision made by an agent in the model concerning salience is made by the incumbent: to commit policy on one particular issue while in office or not. Uncertainty is about the state of nature, which shifts the voter ideal point on the policy-relevant dimension, and is realized after the election. Thus, candidates face uncertainty of voter preferences. Salience occurs when no policy commitment is made, since the issue is relevant for the election. Again, this model is not set up to address the questions we pose.

We take a different approach, allowing candidates to talk about any subset of issues, allowing uncertainty both on the part of voters and candidates, and

\footnotetext{
${ }^{1}$ For most of the paper, candidate ambiguity is exogenous and translation invariant, in the sense that it is represented by the addition of a random variable (with mean zero and variance that is common knowledge) to the position chosen by a candidate or party. In section 5, the incumbent (only) is allowed to choose the variance of their signal subject to a minimum greater than zero. It is unclear whether salience can be generated as an equilibrium in this model due to the constraints imposed on the selection of the variance of the signal.
} 
taking candidates to be office motivated. Once an issue is discussed by a candidate, we assume that they are bound to the position they advocate. Here we also generalize some of the previous literature in a minor way, allowing voters state-dependent components of preferences over candidates that are independent of policy positions.

We assume first that both candidates and voters are expected utility maximizers. Under this assumption, we still find salience results, in that candidates typically want to talk about everything (or they are indifferent between talking and nonsalience). The reason for this salience result is as follows: as long as each candidate is an expected utility maximizer, then a lottery over the set of lotteries that are defined over the set of states can always be translated into a more basic lottery over the set of states. Consider the following example. A candidate is thinking about announcing her position on a risky issue, "gun control." If she does not announce her position, her winning probability is $\frac{1}{2}$. If she announces her position (either for or against), then with probability $\frac{1}{2}$, voters love her policy, and her winning probability increases to $\frac{9}{10}$, while with probability $\frac{1}{2}$, they hate her policy, and her winning probability becomes $\frac{1}{10}$. Assume that the candidate cares only about winning (and thus is a student of Vince Lombardi). Then, under the expected utility hypothesis, she is indifferent between announcing a policy or not, since her overall winning probability in the case of announcement of a policy is still $\frac{1}{2} \cdot \frac{9}{10}+\frac{1}{2} \cdot \frac{1}{10}=\frac{1}{2}$. Thus, in order to obtain robust nonsalience with the idea expressed in our informal story, we need to leave the standard expected utility framework. Candidates should be ambiguity averse players, although voters can be risk neutral expected utility maximizers (unlike Shepsle, 1972).

In the next section, we specify the model and our notation. In section 3, we present the main salience results in our model. In section 4, we present an example using "Knightian uncertainty" or "maxmin expected utility with multiple priors" of Gilboa and Schmeidler (1989) to illustrate how nonsalience of issues might be generated. ${ }^{2}$

\footnotetext{
${ }^{2}$ See Bade (2003) for a very interesting model of elections employing uncertainty aversion to obtain existence of equilibrium in a setting with multiple issues. For an interesting application of ambiguity aversion to a voter's decision in order to study abstention in elections, see Ghirardato and Katz (2002).
} 


\section{The Model}

There are a finite number of agendas or dimensions $\{1, \ldots, A\}$. Two candidates named 1 and 2 , indexed by $i, j($ where $i \neq j$ ), can choose any position between $[-1,1]$ for each agenda $a \in\{1, \ldots, A\} .^{3}$ Candidate $i$ 's strategy is a vector $s^{i}=\left(s_{1}^{i}, \ldots, s_{A}^{i}\right) \in[-1,1]^{A}$. There are a finite number of types of voters $t \in\{1, \ldots, T\}$, each of which has population measure $m^{t}>0$ with $\sum_{t=1}^{T} m^{t}=1$. There are finite number of states, $k \in K=\{1, \ldots, K\}$, and each state $k$ realizes with probability $\pi_{k}$ so that $\sum_{k=1}^{K} \pi_{k}=1$. In each state $k \in K$, voter $t$ 's utility realization from a policy $s=\left(s_{1}, \ldots, s_{A}\right) \in[-1,1]^{A}$ is (a linear utility) $:^{4}$

$$
\bar{U}(t, k ; s)=\sum_{a=1}^{A} s_{a} u_{a}^{t}(k) .
$$

Linear utility is used for two reasons. First, among the set of concave utility functions, it gives ambiguity the best chance. Using another concave utility will only strengthen the results. Second, voters are indifferent between any distribution of policy outcomes and the sure outcome of its mean. Thus, the assumption that an ambiguous strategy yields a payoff of 0 to the voters is the same as assuming that the mean of the ambiguous outcome distribution is 0 . As we shall see in section 4 , this does not imply that the candidate takes a moderate position under Knightian uncertainty.

Assume that each type of voters have preferences over candidates based on the state realized. This is denoted by $u_{i}^{t}(k)$. Thus, in each state $k \in K$, voter $t$ 's

\footnotetext{
${ }^{3}$ Here, we assume that candidates have a continuum of strategies. However, in the case where the candidates' strategy set is $\{-1,1, \emptyset\}$, where $\emptyset$ denotes no announcement, the arguments go through provided that $\emptyset$ generates a payoff of 0 (indeed, this is the case if voters' subjective prior probabilities concerning candidates' positions are .5 on both -1 and 1). If a candidate does not discuss an issue, the position of the candidate is unknown to the voters when they vote, so they use a prior. Linear candidate utility functions are useful here, but since there are only two outcomes of relevance to the candidates (win and lose), risk aversion on the part of candidates would be meaningless. In some of our initial research for this manuscript, we were able to generate nonsalience if voters are risk averse and no announcement yields a certain outcome rather than a lottery. However, this type of model does not seem to be politically meaningful. For example, nothing is ever salient within this framework; see Shepsle (1972).

${ }^{4}$ We use linear utility over the policy space in order to give the best chance for nonsalience to occur in the class of concave utility functions. Obviously, as a corollary of Shepsle (1972), if voters' utility functions are convex, nonsalience may be supported without any problem. However, we strongly doubt the validity of such an assumption (implying that voters prefer candidates who are ambiguous about their policies).
} 
utility realization from candidate $i$ choosing a policy $s=\left(s_{1}, \ldots, s_{A}\right) \in[-1,1]^{A}$ is written as

$$
U(t, k ; s, i)=\bar{U}(t, k ; s)+u_{i}^{t}(k)=\sum_{a=1}^{A} s_{a} u_{a}^{t}(k)+u_{i}^{t}(k) .
$$

Thus, if candidates $i$ and $j$ announce strategies $s^{i}$ and $s^{j}$, type $t$ voters vote for candidate $i$ in state $k$ if

$$
\begin{aligned}
\Delta_{i j} U\left(t, k ; s^{i}, s^{j}\right) & =U\left(t, k ; s^{i}, i\right)-U\left(t, k ; s^{j}, j\right) \\
& =\sum_{a=1}^{A} s_{a}^{i} u_{a}^{t}(k)+u_{i}^{t}(k)-\sum_{a=1}^{A} s_{a}^{j} u_{a}^{t}(k)-u_{j}^{t}(k) \\
& =\sum_{a=1}^{A}\left(s_{a}^{i}-s_{a}^{j}\right) u_{a}^{t}(k)+u_{i}^{t}(k)-u_{j}^{t}(k) \\
& =\Delta_{i j} \bar{U}\left(t, k ; s^{i}, s^{j}\right)+u_{i}^{t}(k)-u_{j}^{t}(k)>0,
\end{aligned}
$$

where $\Delta_{i j} \bar{U}\left(t, k ; s^{i}, s^{j}\right)=\sum_{a=1}^{A}\left(s_{a}^{i}-s_{a}^{j}\right) u_{a}^{t}(k)$. Note that term $u_{i}^{t}(k)-u_{j}^{t}(k)$ simply works as a constant term for each state $k$ : regardless of candidate $i$ 's position, this term is constant. An example would be the competence of the candidate.

When the election takes place, the voters know the state of the world. However, when the candidates choose their strategies, they do not know the state of the world. But the candidates do know the distribution of voter types.

Let $\phi^{i}: T \times K \times[-1,1]^{A} \times[-1,1]^{A} \rightarrow\{0,1\}$ be a support function for candidate $i$ for each type of voter and in each state: i.e., $\phi^{i}\left(t, k, s^{i}, s^{j}\right)=1$ means that voter type $t$ supports candidate $i$ in state $k$ when the strategy profile is $\left(s^{i}, s^{j}\right)$. Thus, $\phi^{i}\left(t, k ; s^{i}, s^{j}\right)=1$ follows if $\sum_{a=1}^{A}\left(s_{a}^{i}-s_{a}^{j}\right) u_{a}^{t}(k)+$ $u_{i}^{t}(k)-u_{j}^{t}(k)>0$. Following the standard tie-breaking rule, let us assume that $\phi^{i}\left(t, k ; s^{i}, s^{j}\right)=\frac{1}{2}$ if $\sum_{a=1}^{A}\left(s_{a}^{i}-s_{a}^{j}\right) u_{a}^{t}(k)+u_{i}^{t}(k)-u_{j}^{t}(k)=0$.

Define the indicator function $I^{i}$, denoting candidate $i$ 's probability of winning in state $k$, as follows.

$$
I^{i}\left(k ; s^{i}, s^{j}\right) \equiv\left\{\begin{array}{c}
1 \text { if } \sum_{t=1}^{T} m^{t} \phi^{i}\left(t, k ; s^{i}, s^{j}\right)>1 / 2 \\
0 \text { if } \sum_{t=1}^{T} m^{t} \phi^{i}\left(t, k ; s^{i}, s^{j}\right)<1 / 2 \\
1 / 2 \text { if } \sum_{t=1}^{T} m^{t} \phi^{i}\left(t, k ; s^{i}, s^{j}\right)=1 / 2
\end{array}\right.
$$

We define $I^{j}$ similarly for candidate $j$ :

$$
I^{j}\left(k ; s^{j}, s^{i}\right) \equiv\left\{\begin{array}{c}
1 \text { if } \sum_{t=1}^{T} m^{t} \phi^{j}\left(t, k ; s^{j}, s^{i}\right)>1 / 2 \\
0 \text { if } \sum_{t=1}^{T} m^{t} \phi^{j}\left(t, k ; s^{j}, s^{i}\right)<1 / 2 \\
1 / 2 \text { if } \sum_{t=1}^{T} m^{t} \phi^{j}\left(t, k ; s^{j}, s^{i}\right)=1 / 2
\end{array}\right.
$$


We can calculate candidate $i$ 's expected winning probability by using the $I^{i}$ function.

$$
w^{i}\left(s^{i}, s^{j}\right)=\sum_{k=1}^{K} \pi_{k} \cdot I^{i}\left(k ; s^{i}, s^{j}\right) .
$$

Similarly,

$$
w^{j}\left(s^{j}, s^{i}\right)=\sum_{k=1}^{K} \pi_{k} \cdot I^{j}\left(k ; s^{j}, s^{i}\right) .
$$

Obviously, $w^{i}\left(s^{i}, s^{j}\right)=1-w^{j}\left(s^{j}, s^{i}\right)$ follows, and for candidates, this voting game is a constant sum game.

Definition 1 A strategy profile $\left(s^{1}, s^{2}\right)$ is a Nash equilibrium if and only if for any $i \in\{1,2\}$, for $j \neq i$, for any $s^{i \prime} \in[-1,1]^{A}, w^{i}\left(s^{i}, s^{j}\right) \geq w^{i}\left(s^{i \prime}, s^{j}\right)$ holds.

\section{Nonsalience is Hard to Generate}

From now on, we assume that "not announcing" policy on dimension $a$ corresponds to position $s_{a}=0 .{ }^{5}$ Given this, we can say the following.

Proposition 1 Suppose that in a Nash equilibrium neither candidate announces a position on agenda $a$. Then, both candidates are indifferent among any positions on agenda $a$. That is, if a Nash equilibrium $\left(s^{1 *}, s^{2 *}\right)$ satisfies $s_{a}^{i *}=s_{a}^{j *}=$ 0 for some $a \in A$, then for any $i \in\{1,2\}$, we have $w^{i}\left(s^{i *}, s^{j *}\right)=w^{i}\left(s_{a}^{\prime}, s_{-a}^{i *}, s^{j *}\right)$ for any $s_{a}^{\prime} \in[-1,1]$.

The implication is that there is a very special balance or symmetry in the distribution of voter preferences. For example, with one issue, one state, and no direct preference over candidates (i.e. $u_{i}^{t}(k)=u_{j}^{t}(k)=0$ ), voters must be split equally (or indifferent) between policies +1 and -1 .

All proofs are contained in the Appendix. Here we give the intuition for the result and the ones that follow. Crucial assumptions for the illustration are that voter utility is linear in the position on an issue taken by a candidate, ${ }^{6}$

\footnotetext{
${ }^{5}$ An interpretation of the model is as follows. The candidates have lexicographic preferences, first over expected probability of winning and then over policy. If a candidate wins, she implements her preferred policy under "no announcement" and the announced policy with an announcement. The latent policy preferences of the candidate are unknown to the voters, but the voters do have a prior over each candidate's ideal policy when no announcement is made by that candidate.

${ }^{6}$ At this point, we wish to remind the reader of the content of footnote 4 . Among concave voter utility functions, linear utility functions give nonsalience the best chance to appear in Nash equilibrium.
} 
and the fact that the candidates play a constant sum game. For the purpose of giving intuition, consider the case of one issue. Please refer to Figure 1 below; the horizontal axis represents the issue space.

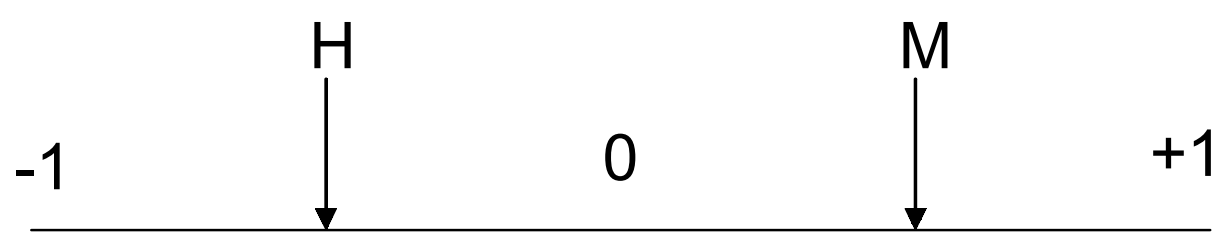

Figure 1: An Illustration

The two candidates are Hideo and Marcus. Suppose that there is a Nash equilibrium with neither candidate announcing, so both are at the position 0 . Obviously, since this is a Nash equilibrium situation, neither candidate can do better in terms of expected winning probability with an alternate strategy. Now suppose that if Marcus shifts his strategy to the right, to the point $M$, and Hideo keeps his strategy at 0, then Marcus does worse than in the Nash equilibrium. Since the game is constant sum, Hideo must do better if Marcus plays strategy M. Note also that since voter utilities are linear, they are translation invariant, so the payoffs to voters would be the same if Hideo plays strategy $\mathrm{H}$ and Marcus plays strategy 0, provided that $\mathrm{H}=-\mathrm{M}$. This means that Hideo could do better playing $\mathrm{H}$ instead of 0 when Marcus plays 0 , so "each player using the 0 strategy" is not an equilibrium, a contradiction.

Remark 1 This claim does not provide a strong statement, but it illustrates the point that we may need Knightian uncertainty on the part of candidates in order to obtain robust nonsalience. ${ }^{7}$

Proposition 2 Let the status quo or the position generated by no announcement be denoted by $s_{a}=S Q_{a} \in[-1,1]$. Suppose that in a Nash equilibrium neither candidate announces a position on agenda $a$. Then, both candidates are

\footnotetext{
${ }^{7}$ It is likely that this result can be pushed further by using weaker assumptions, but since it's a negative result, there seems to be no reason to do so. Note also that we are discussing equilibrium in pure strategies, and that for every equilibrium where nonsalience is the strategy of each candidate, there is an equilibrium where the salience strategy 0 is used by each candidate.
} 
indifferent among any positions in $\left[-1+S Q_{a}, 1\right]$ (if $S Q_{a}>0$ ) or $\left[-1,1+S Q_{a}\right]$ (if $S Q_{a}<0$ ) on agenda a. That is, if a Nash equilibrium $\left(s^{1 *}, s^{2 *}\right)$ satisfies $s_{a}^{i *}=s_{a}^{j *}=S Q_{a}$ for some $a \in A$, then for any $i \in\{1,2\}$, we have $w^{i}\left(s^{i *}, s^{j *}\right)=w^{i}\left(s_{a}^{\prime}, s_{-a}^{i *}, s^{j *}\right)$ for any $s_{a}^{\prime} \in\left[-1+S Q_{a}, 1\right]$ (if $S Q_{a}>0$ ) or $s_{a}^{\prime} \in\left[-1,1+S Q_{a}\right]$ (if $\left.S Q_{a}<0\right)$.

The proof is almost identical to that of Proposition 1.

Proposition 3 Let $S Q_{a}=0$. Suppose that in a Nash equilibrium one candidate $j$ does not announce a position on agenda a, while candidate $i$ announces position $s_{a}^{i *}$. Suppose that $s_{a}^{i *}<0$ (a symmetric argument holds for $s_{a}^{i *}>0$ ). Then, candidate $i$ is indifferent among any positions in $\left[-1,1+s_{a}^{i *}\right]$ on agenda $a$, while candidate $j$ is indifferent among positions $\left[-1-s_{a}^{j *}, 1\right]$. That is, if a Nash equilibrium $\left(s^{1 *}, s^{2 *}\right)$ satisfies $s_{a}^{j *}=0, s_{a}^{i *}<0$ for some $a \in A$, then for any $i \in\{1,2\}$, we have $w^{i}\left(s^{i *}, s^{j *}\right)=w^{i}\left(s_{a}^{\prime}, s_{-a}^{i *}, s^{j *}\right)$ for any $s_{a}^{\prime} \in\left[-1,1+s_{a}^{i *}\right]$, $w^{j}\left(s^{i *}, s^{j *}\right)=w^{j}\left(s_{a}^{\prime}, s_{-a}^{j *}, s^{i *}\right)$ for any $s_{a}^{\prime} \in\left[-1-s_{a}^{i *}, 1\right]$.

Remark 2 These results tell us that nonsalience is unlikely in these games. Of course, we have not said anything about existence of Nash equilibrium in the games, which may be difficult due to the discontinuity in payoff functions. ${ }^{8}$

\section{Nonsalience and Knightian Uncertainty}

We illustrate the possibility of obtaining nonsalience in a Knightian uncertainty framework. We use the tools of Gilboa and Schmeidler (1989). The basic idea is that the utility of a gamble is equal to the minimum expected utility obtained from the gamble taken over a collection of probability distributions (priors or subjective beliefs). Players choose a gamble in order to maximize the minimum expected utility (maximin expected utility with multiple priors). Obviously, when the collection consists of just one element, the theory replicates standard expected utility theory. Let policy space be $Q$, and let a prior be $\pi \in \Delta(Q)$, where $\Delta(\cdot)$ is the space of probability distributions on $\cdot$ The set of priors $M$ is a nonempty convex and (weakly) compact subset of $\Delta(Q)$. Given a

\footnotetext{
${ }^{8}$ See, for example, Ball (1999). He considers a special case of our model, where voters know candidate positions with certainty but candidates are uncertain about voter preferences. Discontinuities can arise whenever candidates are even slightly office motivated. John Duggan has suggested that with the additive, linear utility function we use for candidates, generic existence of a Nash equilibrium where all issues are salient might be proved using linear programming.
} 
(von Neumann-Morgenstern utility) function $u: Q \rightarrow \Re$, a minimum expected utility under the set of priors $M$ is $U(M)=\min _{\pi \in M} \int u d \pi$, or if $Q$ is a finite set, $U(M)=\min _{\pi \in M} \sum_{q \in Q} \pi(q) u(q)$. Thus, a minimum expected utility is an expected utility under the worst case scenario from the set of possible priors. We apply this to the candidates in our model..$^{9}$

Let there be only one agenda $(A=1)$ with the strategy set of candidates taken to be $S=\{-1,1, \emptyset\}$ (here $\emptyset$ is taken to mean "no announcement"; see footnote 1$)$, one type of voter $(T=1)$, and four states with two dimensions

$$
K=\left\{\left(r_{1}, a_{-1}\right),\left(r_{1}, a_{+1}\right),\left(r_{2}, a_{-1}\right),\left(r_{2}, a_{+1}\right)\right\}
$$

Here, one dimension $K^{r}=\left\{r_{1}, r_{2}\right\}$ is a purely random factor in voters' preferences over candidates and the other dimension $K^{a}=\left\{a_{-1}, a_{+1}\right\}$ is a random factor based on preferences over policy. We assume that the distributions of $r$ and $a$ are statistically independent with respect to each other. We assume that the probabilities of occurrence of $r_{1}$ and $r_{2}$ are each $\frac{1}{2}$. These probabilities are objectively known to the candidates (or candidates have these subjective beliefs), but candidates have ambiguous beliefs over the occurrences of $a_{-1}$ and $a_{+1}$; each occurs with probability between $\frac{1}{3}$ and $\frac{2}{3}$. That is, the set of subjective beliefs of $a_{1}$ occurring is $\left[\frac{1}{3}, \frac{2}{3}\right]$. Also, let $u_{i}\left(r_{i}\right)=\epsilon>0$ and $u_{i}\left(r_{j}\right)=0$ for any $i, j=1,2$ with $i \neq j$, and let $v\left(a_{-1},-1\right)=v\left(a_{+1},+1\right)>2 \epsilon$, and $v\left(a_{-1},+1\right)=v\left(a_{+1},-1\right)=0 . \quad$ For $(r, a, s) \in K \times S, U(i, r, a ; s)=$ $u_{i}(r)+v(a, s) \cdot{ }^{10} \quad$ Given these utility functions, candidates know that if an agenda is announced, then voters' tastes over policy dictate the outcome (tastes over candidates do not matter). However, candidates have ambiguous beliefs over voters' tastes over policy. But they are certain that voters' tastes over candidates are evenly split. Thus, candidates prefer nonsalience under ambiguity.

Indeed, if candidate $i$ announces +1 , then she loses if the state realization is either $\left(r_{1}, a_{-1}\right)$ or $\left(r_{2}, a_{-1}\right)$, and wins if the state realization is either $\left(r_{1}, a_{+1}\right)$ or $\left(r_{2}, a_{+1}\right)$. However, her maximum subjective probability of losing is $\frac{2}{3}$, and as a result, her maximin payoff is $\frac{1}{3} \cdot 1+\frac{2}{3} \cdot(0)=\frac{1}{3}$. On the other hand, if she does not announce her position, then her maximin payoff is $\frac{1}{2} \cdot 1+\frac{1}{2} \cdot(0)=\frac{1}{2}$. Therefore,

\footnotetext{
${ }^{9}$ Of course, whether candidates (or more generally, people) in the real world are ambiguity averse is an empirical question. Experimental evidence suggests they might be; see Tversky and Heath (1991), Maffioletti and Santoni (2000), Chow and Sarin (2001), and Ho et al (2002).

${ }^{10}$ Notice that this specification of voter utility differs slightly from the specification given in section 2 , since it is not symmetric around 0 . We do this to make calculations easy.
} 
she has no incentive to announce policy given a nonsalience situation.

In this example, we assumed that the two candidates are symmetric in terms of $u_{i}(r)$. This is the reason why a candidate's winning probability under nonsalience is exactly $\frac{1}{2}$. Obviously, we can make it asymmetric. As long as both candidates' winning probabilities under nonsalience are more than $\frac{1}{3}$, nonsalience is the only equilibrium of this voting game. However, if a candidate has a winning probability under nonsalience of less than $\frac{1}{3}$, she is better off announcing her position. In such a case, her opponent wants to announce the same policy as hers in order to cancel the ambiguity effects out. Thus, the game has the same structure as the classical game of "matching pennies", and equilibrium necessarily implies mixed strategy plays in this specific example. ${ }^{11}$

Now let us return to the question raised in the introduction concerning the equivalence between some policy announcement and no announcement. As long as there is no strategy involving an announcement of policy available to candidates that yields an outcome independent of the states $a_{+1}$ and $a_{-1}$, there is no announcement strategy that will yield the same distribution of payoffs as "keeping quiet" in this structure.

What can we conclude from this example? With some ambiguity in priors, but not too much, nonsalience is possible in the framework of Knightian uncertainty. Even with a great deal of ambiguity, if a candidate is an apparent underdog then salience reappears, and candidates announce positions. Consider an example where Bush and Gore are the two candidates and there is no apparent underdog. Suppose that nonsalience on gun control appears (assuming that they feel a great deal of ambiguity over voters' preferences on gun control). Even if Gore may be expecting that his chance of winning is $45 \%$, he still does not want to discuss the issue. However, if the two candidates are Buchanan and Bush, and Buchanan does not have a good chance to win the race, he is happy to announce his position. Then, in order to eliminate Buchanan's possibility of winning due to luck, Bush now wants to announce the same position as Buchanan so that voters cannot distinguish between their policies. $^{12}$

\footnotetext{
${ }^{11}$ Steve Callander has pointed out that it might be possible to derive this framework as a reduced form of a bigger, structural game where candidates are expected utility optimizers. In this case, there are some other agents (such as the press or opposition legislators) who play a zero sum game with the candidates and try their best to embarrass or weaken each candidate by raising the issues. Each issue would have a different prior associated with it, their union generating the collection $\Delta$. In this game, the candidates might play maximin.

${ }^{12}$ In reality, part of the ambiguity would be resolved immediately after Buchanan an-
} 


\section{$5 \quad$ Discussion and Extensions}

In this section, we discuss our approach and possible extensions. ${ }^{13}$ First of all, even under Knightian uncertainty, nonsalience is not the only equilibrium. In the basic example in the previous section, both candidates may announce the same position in an equilibrium, since it removes ambiguity from both candidates' calculations. A little bit more formally, we can show that neither candidate has an incentive to deviate from this strategy profile unilaterally. A candidate does not change her position, since it creates a big ambiguity in outcome, and her worst winning probability becomes $\frac{1}{3}$ again. She also does not want to choose not to announce, since this way her winning probability again becomes ambiguous, and the worst case for her (the best case for the other candidate) is winning with probability $\frac{1}{3}$. In both cases, the worst winning probability is less than the winning probability $\frac{1}{2}$ generated by announcing the same position.

Moreover, if there are many risky (ambiguous) issues (with ex ante voters' distribution more or less split at 50/50), then there are many equilibria as well. A strategy profile in which both candidates announce the same policies on any subset of risky agendas or dimensions is an equilibrium. This result may sound discouraging, but it is at least interesting to see that both candidates would announce the same policies on ambiguous agendas. This may be interpreted as a Downsian theorem in a multidimensional issue space, although the equilibrium policy is not necessarily at the center.

We can introduce a little bit of dynamics into the model. One possible reason why candidates do not announce their positions on risky issues may be the following. If a candidate announces her policy on a risky issue, then she loses if voters happen to hate her policy. Her best case scenario is obviously the case that the voters like her policy. However, once such information is revealed, then the other candidate may follow to announce the same position. Of course, the follower's policy may be discounted by voters, but if voters like the follower more than the leader in other respects, then the first mover might still lose in the election. This implies that the return from announcing a policy first is

nounces his policy. Bush can wait to see what polls say about gun control, and he can then decide if he announces "for" or "against" on this issue.

${ }^{13}$ It might be interesting to examine a model where candidates maximize expected probability of winning, but voters are ambiguity averse. Our purpose in this paper was to generate a tractable model that requires the least deviation from expected utility theory but generates robust salience and nonsalience. 
less than in the simultaneous move game. Thus, if we allow sequential moves, then the incentives for candidates to announce their positions on risky issues can be significantly reduced. In such a case, many salience equilibria where candidates announce the same policies, discussed in the previous paragraph, can be eliminated.

The discussion in the preceding paragraph bears a superficial resemblance to the discussion in Glazer (1990, p. 240). There are two real differences. First, as we have noted, the models are formally distinct. Second, in Glazer's model, "In a Nash equilibrium, therefore, no candidate would be the first to announce a position...", while in our model, it might still be the case that a candidate will announce a position first, provided that they are an underdog. This potentially testable hypothesis distinguishes the two models.

Another possibility is to add voter turnout in elections. Intuitively, our informal story to support nonsalience fits in with the issues related to voter turnout. For example, consider the case where gun control is a risky issue, and many voters care about it a lot but not about other issues. Assume that voting is costly. In such a case, if candidates do not announce policies on gun control, then many voters do not turn out, since their benefits from voting are low. If candidates have good information about voters who turn out for other issues, yet they know very little about preferences of voters who turn out when gun control is salient, then they may avoid announcing their policies on gun control. Such a policy announcement simply makes the election result more unpredictable. Thus, ambiguity-averse candidates prefer nonsalience on the issue of gun control. In this paper, we did not adopt this attractive story only because we could not find a nice theory of voter turnout. Even if such a theory were available, the melding of the two models might prove intractable.

Finally, we discuss the evaluation of the Nash equilibria in terms of welfare. Notice that in the case of standard expected utility maximizing candidates as well as equilibrium with minimum expected utility maximizing candidates, the expected probabilities of winning sum to 1 . So candidate welfare is optimized. Moreover, if in every state of nature and for each issue there is a type of consumer who prefers +1 and another who prefers -1 , then all outcomes (not just equilibrium outcomes) are in the Pareto set. 


\section{References}

[1] Adams, J., 1999, Multiparty Spatial Competition with Probabilistic Voting, Public Choice 99, 259-274.

[2] Alesina, A. and A. Cukierman, 1990, The Politics of Ambiguity, The Quarterly Journal of Economics 105, 829-850.

[3] Aragones, E. and A. Postlewaite, 2002, Ambiguity in Election Games, Review of Economic Design 7, 233-255.

[4] Bade, S., 2003, Electoral Competition with Uncertainty Averse Parties, working paper.

[5] Ball, Richard, 1999, Discontinuity and Non-Existence of Equilibrium in the Probabilistic Spatial Voting Model, Social Choice and Welfare 16, 533-555.

[6] Chow, C.C. and R.K. Sarin, 2001, Comparative Ignorance and the Ellsberg Paradox, Journal of Risk and Uncertainty 22, 129-139.

[7] Ghirardato, P. and J.N. Katz, 2002, Indecision Theory: Quality of Information and Voting Behavior, California Institute of Technology Social Science Working Paper 1106R.

[8] Gilboa, I. and D. Schmeidler, 1989, Maxmin Expected Utility with Nonunique Prior, Journal of Mathematical Economics 18, 141-53.

[9] Glazer, A., 1990, The Strategy of Candidate Ambiguity, The American Political Science Review 84, 237-241.

[10] Glazer, A. and S. Lohmann, 1999, Setting the Agenda: Electoral Competition, Commitment of Policy, and Issue Salience, Public Choice 99, 377-394.

[11] Heath, C. and A. Tversky, 1991, Preference and Belief: Ambiguity and Competence in Choice under Uncertainty, Journal of Risk and Uncertainty $4,5-28$.

[12] Ho, J.L.Y., L.R. Keller and P. Keltyka, 2002, Effects of Outcome and Probabilistic Ambiguity on Managerial Choices, Journal of Risk and Uncertainty 24, 47-74. 
[13] Maffioletti, A. and M. Santoni, 2000, Do Trade Union Leaders Exhibit Ambiguity Reaction?, Rivista Internazionale di Scienze Sociali 108, 357376.

[14] McKelvey, R., 1980, Ambiguity in Spatial Models of Policy Formation, Public Choice 35, 385-402.

[15] Shepsle, K.A., 1972, The Strategy of Ambiguity: Uncertainty and Electoral Competition, The American Political Science Review 66, 555-568. 


\section{APPENDIX 1: Proofs}

Proof of Proposition 1: Since $\left(s^{1 *}, s^{2 *}\right)$ is a Nash equilibrium, for any $i \in\{1,2\}, w^{i}\left(s^{i *}, s^{j *}\right) \geq w^{i}\left(s_{a}^{\prime}, s_{-a}^{i *}, s^{j *}\right)$ holds for any $s_{a}^{\prime} \in[-1,1]$. Thus, we only need to show that $w^{i}\left(s^{i *}, s^{j *}\right)>w^{i}\left(s_{a}^{\prime}, s_{-a}^{i *}, s^{j *}\right)$ cannot happen for any $s_{a}^{\prime} \in[-1,1]$. Suppose that for some $s_{a}^{\prime} \neq 0$, it does. We show that in such a case candidate $j$ has an incentive to switch her position on dimension $a$. Note that at state $k$, a type $t$ voter's utility increases when this person votes for $i$ instead of $j$. The utility differences are

$$
\begin{aligned}
\Delta_{i j} \bar{U}\left(t, k ; s^{i *}, s^{j *}\right) & =\sum_{a^{\prime}=1}^{A}\left(s_{a^{\prime}}^{i *}-s_{a^{\prime}}^{j *}\right) u_{a^{\prime}}^{t}(k) \\
& =\left(s_{a}^{i *}-s_{a}^{j *}\right) u_{a}^{t}(k)+\sum_{a^{\prime} \neq a}\left(s_{a^{\prime}}^{i *}-s_{a^{\prime}}^{j *}\right) u_{a^{\prime}}^{t}(k) \\
& =\sum_{a^{\prime} \neq a}\left(s_{a^{\prime}}^{i *}-s_{a^{\prime}}^{j *}\right) u_{a^{\prime}}^{t}(k),
\end{aligned}
$$

and

$$
\begin{aligned}
\Delta_{i j} \bar{U}\left(t, k ; s_{a}^{\prime}, s_{-a}^{i *}, s^{j *}\right) & =\left(s_{a}^{\prime}-s_{a}^{j *}\right) u_{a}^{t}(k)+\sum_{a^{\prime} \neq a}\left(s_{a^{\prime}}^{i *}-s_{a^{\prime}}^{j *}\right) u_{a^{\prime}}^{t}(k) \\
& =s_{a}^{\prime} u_{a}^{t}(k)+\sum_{a^{\prime} \neq a}\left(s_{a^{\prime}}^{i *}-s_{a^{\prime}}^{j *}\right) u_{a^{\prime}}^{t}(k) \\
& =s_{a}^{\prime} u_{a}^{t}(k)+\Delta_{i j} \bar{U}\left(t, k ; s^{i *}, s^{j *}\right) .
\end{aligned}
$$

Now, consider the case where candidate $i$ does not change her policy yet candidate $j$ announces $-s_{a}^{\prime}$ instead of $s_{a}^{j *}=0$. Then, by the same logic as before, for any $t$ and $k$, we have

$$
\begin{aligned}
\Delta_{j i} \bar{U}\left(t, k ;-s_{a}^{\prime}, s_{-a}^{j *}, s^{i *}\right) & =-s_{a}^{\prime} u_{a}^{t}(k)+\sum_{a^{\prime} \neq a}\left(s_{a^{\prime}}^{j *}-s_{a^{\prime}}^{i *}\right) u_{a^{\prime}}^{t}(k) \\
& =-s_{a}^{\prime} u_{a}^{t}(k)+\Delta_{j i} \bar{U}\left(t, k ; s^{j *}, s^{i *}\right) \\
& =-s_{a}^{\prime} u_{a}^{t}(k)-\Delta_{i j} \bar{U}\left(t, k ; s^{i *}, s^{j *}\right) \\
& =-\Delta_{i j} \bar{U}\left(t, k ; s_{a}^{\prime}, s_{-a}^{i *}, s^{j *}\right) \\
& =\Delta_{j i} \bar{U}\left(t, k ; s^{j *}, s_{a}^{\prime}, s_{-a}^{i *}\right) .
\end{aligned}
$$

Hence,

$$
\Delta_{j i} U\left(t, k ;-s_{a}^{\prime}, s_{-a}^{j *}, s^{i *}\right)=\Delta_{j i} U\left(t, k ; s^{j *}, s_{a}^{\prime}, s_{-a}^{i *}\right) .
$$

In other words, consider the utility difference between candidates $j$ and $i$ for any voter. The difference (between the candidates) in voter utility in the case 
where candidate $j$ announces $-s_{a}^{\prime}$ instead of $s_{a}^{j *}=0$ is the same as the difference (between the candidates) in voter utility from candidate $i$ announcing $s_{a}^{\prime}$ instead of $s_{a}^{*}$. Therefore, voters choosing one candidate in the first case will choose the same candidate in the second case. Thus,

$$
\phi^{j}\left(t, k ;-s_{a}^{\prime}, s_{-a}^{j *}, s^{i *}\right)=\phi^{j}\left(t, k ; s^{j *}, s_{a}^{\prime}, s_{-a}^{i *}\right) \forall t, \forall k
$$

and

$$
I^{j}\left(k ;-s_{a}^{\prime}, s_{-a}^{j *}, s^{i *}\right)=I^{j}\left(k, s^{j *}, s_{a}^{\prime}, s_{-a}^{i *}\right) \forall k
$$

Multiplying by $\pi_{k}$ and summing over states $k$,

$$
\begin{aligned}
w^{j}\left(-s_{a}^{\prime}, s_{-a}^{j *}, s^{i *}\right) & =w^{j}\left(s^{j *}, s_{a}^{\prime}, s_{-a}^{i *}\right) \\
& =1-w^{i}\left(s_{a}^{\prime}, s_{-a}^{i *}, s^{j *}\right)
\end{aligned}
$$

Therefore, we can conclude

$$
w^{j}\left(-s_{a}^{\prime}, s_{-a}^{j *}, s^{i *}\right)=1-w^{i}\left(s_{a}^{\prime}, s_{-a}^{i *}, s^{j *}\right)>1-w^{i}\left(s^{i *}, s^{j *}\right)=w^{j}\left(s^{j *}, s^{i *}\right) .
$$

This is a contradiction to our supposition that $\left(s^{i *}, s^{j *}\right)$ is a Nash equilibrium. Thus, for any $i \in\{1,2\}$, we must have $w^{i}\left(s^{i *}, s^{j *}\right)=w^{i}\left(s_{a}^{\prime}, s_{-a}^{i *}, s^{j *}\right)$ for any $s_{a}^{\prime} \in[-1,1]$.

Proof of Proposition 3: Since $\left(s^{1 *}, s^{2 *}\right)$ is a Nash equilibrium, for any $i \in\{1,2\}, w^{i}\left(s^{i *}, s^{j *}\right) \geq w^{i}\left(s_{a}^{\prime}, s_{-a}^{i *}, s^{j *}\right)$ holds for any $s_{a}^{\prime} \in[-1,1]$. Thus, we only need to show that $w^{i}\left(s^{i *}, s^{j *}\right)>w^{i}\left(s_{a}^{\prime}, s_{-a}^{i *}, s^{j *}\right)$ cannot happen for any $s_{a}^{\prime} \in\left[-1,1+s_{a}^{i *}\right]$. Suppose that for some $s_{a}^{\prime}$, it does. We show that in such a case candidate $j$ has an incentive to switch her position on dimension $a$. Now, consider the case where candidate $j$ does not change her policy yet candidate $i$ announces $s_{a}^{\prime}$ instead of $s_{a}^{i *}$. Then, by the same logic as before, for any $t$ and $k$, we have

$$
\begin{aligned}
\Delta_{i j} \bar{U}\left(t, k ; s_{a}^{\prime}, s_{-a}^{i *}, s^{j *}\right) & =s_{a}^{\prime} u_{a}^{t}(k)+\sum_{a^{\prime} \neq a}\left(s_{a^{\prime}}^{i *}-s_{a^{\prime}}^{j *}\right) u_{a^{\prime}}^{t}(k) \\
& =\left(s_{a}^{i *}-\left\{s_{a}^{i *}-s_{a}^{\prime}\right\}\right) u_{a}^{t}(k)+\sum_{a^{\prime} \neq a}\left(s_{a^{\prime}}^{i *}-s_{a^{\prime}}^{j *}\right) u_{a^{\prime}}^{t}(k) \\
& =\Delta_{i j} \bar{U}\left(t, k ; s^{i *},\left\{s_{a}^{i *}-s_{a}^{\prime}\right\}, s_{-a}^{j *}\right)
\end{aligned}
$$

Hence,

$$
\Delta_{i j} U\left(t, k ; s_{a}^{\prime}, s_{-a}^{i *}, s^{j *}\right)=\Delta_{i j} U\left(t, k ; s^{i *},\left\{s_{a}^{i *}-s_{a}^{\prime}\right\}, s_{-a}^{j *}\right) .
$$


In other words, consider the utility difference between candidates $i$ and $j$ for any voter. The difference (between the candidates) in voter utility in the case where candidate $i$ announces $s_{a}^{\prime}$ instead of $s_{a}^{i *}=0$ is the same as the difference (between the candidates) in voter utility from candidate $j$ announcing $\left\{s_{a}^{i *}-s_{a}^{\prime}\right\}$ instead of $s_{a}^{j *}$. Therefore, voters choosing one candidate in the first case will choose the same candidate in the second case. Thus,

$$
\phi^{j}\left(t, k ; s^{j *}, s_{a}^{\prime}, s_{-a}^{i *}\right)=\phi^{j}\left(t, k ;\left\{s_{a}^{i *}-s_{a}^{\prime}\right\}, s_{-a}^{j *}, s^{i *}\right) \forall t, \forall k
$$

and

$$
I^{j}\left(k ; s^{j *}, s_{a}^{\prime}, s_{-a}^{i *}\right)=I^{j}\left(k,\left\{s_{a}^{i *}-s_{a}^{\prime}\right\}, s_{-a}^{j *}, s^{i *}\right) \forall k
$$

Multiplying by $\pi_{k}$ and summing over states $k$,

$$
\begin{aligned}
w^{j}\left(\left\{s_{a}^{i *}-s_{a}^{\prime}\right\}, s_{-a}^{j *}, s^{i *}\right) & =w^{j}\left(s^{j *}, s_{a}^{\prime}, s_{-a}^{i *}\right) \\
& =1-w^{i}\left(s_{a}^{\prime}, s_{-a}^{i *}, s^{j *}\right)
\end{aligned}
$$

Therefore, candidate $j$ could obtain the same effect against equilibrium strategy $s^{i *}$ by announcing position $\left\{s_{a}^{i *}-s_{a}^{\prime}\right\}$ for issue $a$. We can conclude

$$
\begin{aligned}
w^{j}\left(\left\{s_{a}^{i *}-s_{a}^{\prime}\right\}, s_{-a}^{j *}, s^{i *}\right) & =w^{j}\left(s^{j *}, s_{a}^{\prime}, s_{-a}^{i *}\right) \\
& =1-w^{i}\left(s_{a}^{\prime}, s_{-a}^{i *}, s^{j *}\right) \\
& >1-w^{i}\left(s^{i *}, s^{j *}\right) \\
& =w^{j}\left(s^{j *}, s^{i *}\right) .
\end{aligned}
$$

This is a contradiction to our supposition that $\left(s^{i *}, s^{j *}\right)$ is a Nash equilibrium. Thus, for any $i \in\{1,2\}$, we must have $w^{i}\left(s^{i *}, s^{j *}\right)=w^{i}\left(s_{a}^{\prime}, s_{-a}^{i *}, s^{j *}\right)$ for any $s_{a}^{\prime} \in\left[-1,1+s_{a}^{i *}\right]$. 


\section{APPENDIX 2: An Example of Glazer (1990)}

In the notation of Glazer (1990), $s \in S$ is a candidate position, where $S \subseteq \Re$. Voters have strictly single peaked utility functions, with the median voter's peak normalized to 0. Glazer (1990, p. 238) states:

More formally, let the median voter believe with probability $V(s)$ the candidate's position is to the left of point $s$. The corresponding probability density is $v(s)$. Let $C(s)$ be the probability that the distance between the candidate's announced position and the median voter's ideal point is $s$. (The distance is positive if the candidate's announced position is to the right of the median voter's ideal point and negative if it lies to the left.) The corresponding probability density function is $c(s)$. Notice that if the candidate had perfect information, $C(s)$ would collapse around zero.

Please note that $v(s)$ is the voter's belief that the difference between the candidate position and the voter ideal point is $s$, while $c(s)$ is gives the candidate's belief that the difference between the candidate's position and the voter's ideal point is $s$.

In order to clarify matters, it is easiest to proceed with an example rather than an abstract discussion. We provide detail of the second example in Glazer (1990). ${ }^{14}$ It begins halfway down the right column of p. 239.

For example, let $s$ take on only values $-k,-1,0,1$, and $k$, where $k>1$; and let $c(s)=v(s)=1 / 5$ for each of these values. Let the median voter's utility function be $-s^{2}$. The voter's expected utility from seeing the election of a candidate with an ambiguous position is $-\frac{2}{5}\left(k^{2}+1\right)$. With probability $\frac{3}{5}$ a candidate who specifies a position chooses a position within one unit of the median voter's ideal point. If $|-1|<\left|-\frac{2}{5}\left(k^{2}+1\right)\right|$, that is, if $k>\sqrt{\frac{3}{2}}$, a candidate who specifies a position will defeat an ambiguous candidate with probability of three-fifths. If, however, $k<\sqrt{\frac{3}{2}}$, a candidate who specifies a position will defeat an ambiguous candidate only if the former announced a position coincident with the median voter's ideal point. Since by assumption this occurs only with probability

\footnotetext{
${ }^{14}$ There are examples with discrete and continuous distributions in Glazer (1990). We choose the discrete example to keep calculations simple.
} 
one-fifth, ambiguity would be the dominant strategy. Thus, a Nash equilibrium could have both candidates ambiguous, even though voters are risk averse.

The last result is clearly in conflict with our theorems, so it is important to delineate precisely the difference in the models. As there are no formal theorems and little formal structure in Glazer (1990), in this exercise it is vital to write down a Bayesian game consistent with Glazer's framework. There are three immediate hazards that must be addressed. First, the range of both the median voter ideal point and the candidate position must be unbounded, for otherwise there are endpoint problems in that the maximal and minimal values for ideal points and positions do not have the specified conditional distributions. Second, in order to have a "consistent" belief system for the Bayesian game, we must find a joint distribution on player types (two candidate positions and a median voter ideal point) that justifies these conditional distributions. Finally, $k$ can be any real number, so the set of admissible ideal points and candidate positions might not be closed under addition or subtraction. ${ }^{15}$

To address the first challenge, we allow any integer as a candidate position or median voter ideal point.

To address the third challenge, we simplify the model a bit: we assume that each candidate's position can be only $-1,0$, or 1 relative to the median voter's position of $0 .{ }^{16}$ The conditional probability is $\frac{1}{3}$ each. Given this distribution, the median voter's utility from ambiguous candidate is $\frac{1}{3} \cdot(-1)+\frac{1}{3} \cdot 0+\frac{1}{3}$. $(-1)=-\frac{2}{3}>-1 .{ }^{17}$ Thus, an ambiguous candidate is more attractive than

\footnotetext{
${ }^{15}$ Thus, if $k$ is not an integer, we cannot construct a consistent belief system (see the second point).

${ }^{16}$ Note that we can modify Glazer's example keeping five possible positions. We set $k=2$. Obviously, if we keep the uniform conditional distribution, ambiguity is not the best response to an ambiguous opponent as Glazer points out (this case corresponds to $k>\sqrt{\frac{3}{2}}$ ). We can recover ambiguity in equilibrium by modifying the median voter's belief. Let $p$ be the conditional probability that the candidate's position is extreme $(-2$ or 2$)$ relative to the median voter ideal point : more specifically, let the belief over relative positions $-2,-1,0,1$, and 2 be $\frac{p}{2}, \frac{1-p}{3}, \frac{1-p}{3}, \frac{1-p}{3}, \frac{p}{2}$, respectively. Given this distribution, the median voter's utility from an ambiguous candidate is $2 \cdot(-2) \cdot \frac{p}{2}+2 \cdot(-1) \cdot \frac{1-p}{3}=-2 p-\frac{2}{3}(1-p)=-\frac{2}{3}-\frac{4}{3} p$. Thus, if $-\frac{2}{3}-\frac{4}{3} p>-1$ (or $p<\frac{1}{4}$ ), then an ambiguous candidate is more attractive than the candidate whose position is not 0 . This implies that if $p<\frac{1}{4}$ then making a position ambiguous is an ex ante dominant strategy.

${ }^{17}$ This is obviously true in Glazer's case, too. Thus, we only need three possible (relative) positions for a policy ambiguity result. It is our guess that Glazer introduced five points in order to make the desirability of policy ambiguity dependent on the parameter $k$.
} 
the candidate whose announced position is not 0 . This implies that making policy ambiguous is always an ex ante dominant strategy.

The domain of non-zero probability assigned to the joint distribution of median voter ideal points and one of the candidates is given in Figure 2.

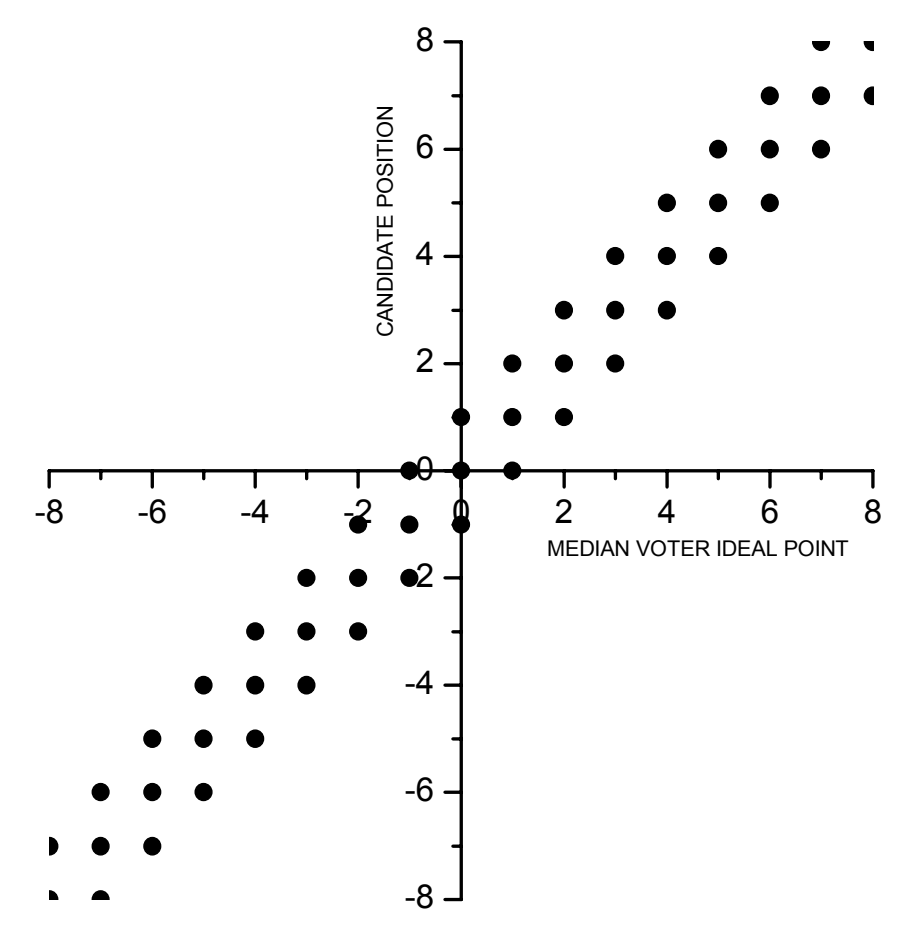

Figure 2: A Variant of Glazer's Example

This is what is required by the assigned conditional distributions. A median voter ideal point of 0 allows candidate positions of $-1,0$, and 1 . In the end, the conditional distribution for the median voter must be $\frac{1}{3}$ each. Similarly, a candidate position of 0 allows median voter ideal points of $-1,0$, and 1 . In the end, the conditional distribution must be $\frac{1}{3}$ each.

Turning next to the second challenge, we must find a joint distribution on the two candidates' positions and the median voter's ideal point that will generate the appropriate conditional distributions for every realization. Obviously, we cannot assign uniform unconditional probability to all permissible configurations of the three random variables, since their support is unbounded and thus zero probability must be assigned to each realization. However, we can almost do it as follows, and this is good enough for our purposes. Fix 
$\delta>0, \delta<1$. Give the random variable $\alpha$ of the ideal point for the median voter the distribution over the integers that assigns probability $\delta^{|k|} \cdot \frac{1-\delta}{1+\delta}$ to integer $k$. Then the maximal ratio between the mass assigned to two integers whose difference is at most 2 is $\delta^{2}$. It isn't perfect, but it's as close to uniform as we need. Let $\phi$ and $v$ be random variables that are mutually stochastically independent and each independent of $\alpha$ that place mass $\frac{1}{3}$ on each of $-1,0$, and 1. Define $\beta=\alpha+\phi$, and $\gamma=\alpha+v$. If the median voter gets private information $\alpha$, one candidate gets private information $\beta$ and the other candidate gets private information $\gamma$, then the conditional distributions of the candidates are as specified by Glazer. For every $\epsilon>0$ we can find a positive $\delta<1$ but sufficiently close to 1 such that the conditional distribution of the median voter is within $\epsilon$ of a uniform distribution. ${ }^{18}$

Now that we have a properly specified Bayesian game with consistent system of beliefs, we can return to the conflict between our results and Glazer's: can ambiguity arise as a robust equilibrium strategy? The crucial argument, dating back to Shepsle (1972), is as follows. If voters are risk averse and ambiguity generates risk to the voters in the form of a nondegenerate distribution over policy outcomes, and a candidate can choose to announce the mean of this distribution as her policy, then announcing this policy weakly (second order) stochastically dominates ambiguity and thus the voters prefer it. In our model, this is true. In Glazer's model, the candidates cannot choose to announce the mean of the median voter's conditional distribution over policy outcomes under ambiguity, since they don't know where it is. They can only announce $\beta$ or $\gamma$, and each of these is at the mean of the distribution (i.e. at $\alpha$ ) with probability only $\frac{1}{3}$. This is the difference in assumptions. In our view, it is not unreasonable for the candidates to acquire information on the mean of the median voter's view of the candidate's position under no announcement through survey data. ${ }^{19}$

\footnotetext{
${ }^{18}$ Thus, if $\epsilon$ is close to zero, we can almost make both $v(s)$ and $c(s)$ uniform at $\frac{1}{3}$ (Glazer's notation).

${ }^{19}$ Ken Binmore and John Ledyard have encouraged us to extend this example to general atomless distributions. Our conjecture is that with enough freedom in the choice of distribution, we can generate just about anything as an equilibrium in the static model.
} 